\title{
Impact of Spatial Visualization Aptitude on WWW Navigation ${ }^{\S}$
}

\author{
James Blustein", Ishtiaq Ahmed, Haris Parvaiz, Ching-Lung Fu, Caixia Wang, \\ Alexander (Sandy) Chapman and Yeming Hu
}

Faculty of Computer Science Dalhousie University 6050 University Avenue Halifax, Nova Scotia B3H 1W5, Canada

\begin{abstract}
Although the underlying mechanism is not well understood, there is considerable evidence that the constellation of cognitive factors known as 'spatial aptitude' influences users' performance in information spaces. Evidence of the effect in the computer science literature is contradictory: some studies show that techniques, which support users with lower aptitude, retard performance by those with higher aptitude. We have investigated the effect of the visualization subfactor in a real-world navigation task using location menu breadcrumbs and Dillon's IMRD task.

We compared the navigational styles and success rates in an answer seeking task using both standard and menu breadcrumbs in a large website. The higher aptitude group was significantly more efficient and used the Back button less than the lower aptitude group.

We discuss implications for explaining why spatial aptitude affects success with hypertext, the potential for practical application, and ongoing follow-up work.
\end{abstract}

Keywords: Hypertext, navigation, cognitive factors, WWW, adaptive hypermedia, spatial ability/aptitude, information architecture, information shape, performance of document systems.

\section{INTRODUCTION}

This article is about the interaction of spatial aptitude and success in using hypertext that is not similar to navigating in the physical world. Although we include hypermedia in our working definition of hypertext, we are studying presentations that are primarily text-based.

There is a long tradition of trying to understand the fundamental reasons that hypertext succeeds (or does not) [1-3]. The so-called lost-in-hyperspace phenomenon is a well-known hindrance to achieving the potential of hypertext to improve the human condition. The phenomenon is now often termed 'the navigation problem' presupposing both a cause and solution.

In open problems, such as navigation, where people must form plans as part of their solutions, individual differences are often a likely predictor of success $[1,4]$. Chen and Rada [5] were surprised to find, in their meta-analysis of 23 experiments, that so-called 'spatial ability' was the only individual difference that had a measurable effect on success with hypertext (in terms of efficiency or effectiveness).

We chose the further investigate the role of spatial aptitude on styles of, and success in, hypertext navigation. We use a large hierarchically structured website to make the study realistic and relevant as such websites abound. A further advantage of our selection is that key properties of navigation in such structures are well known [6-8]. A note

*Address correspondence to this author at the Faculty of Computer Science Dalhousie University 6050 University Avenue, Halifax, Nova Scotia, B3H 1W5, Canada; E-mail: Jamie@cs.dal.ca

${ }^{\S}$ This article is a revision and expansion of an earlier article that was requested by the editors for submission for consideration in a special issue. about terminology is needed here: What is now known as spatial aptitude (SA) used to be called 'spatial ability', however since it is now recognized that it can be changed through practice and study, the term aptitude is used instead of ability.

This work is in two parts: (1) an analysis of spatial aptitude data collected as part of an experiment presented earlier [9]; and (2) an outline of our ongoing follow-ups to that study.

\subsection{Spatial Visualization Aptitude}

Spatial aptitude is the property of mind that requires several psychological attributes such as aptitude to encode information, remember, transform and differentiate spatial objects [10]. Carroll [11] conducted an exploratory survey that yielded five factors of spatial aptitude belonging to the visual perceptual domain: visualization (VZ), spatial relations (SR), closure speed (CS), closure flexibility (CF), and perceptual speed (PS). Spatial visualization has been found as the most important factor among the factors of spatial aptitude that influence navigation in the WWW [1113]. SV is the 'ability in manipulating visual patterns, as indicated by level of difficulty and complexity in visual stimulus material that can be handled successfully, without regard to the speed of task solution' [11] (p. 362). Throughout the rest of this article, the term spatial aptitude refers to the constellation of individual's psychological measurements of VZ. Spatial visualization requires either the mental restructuring of a figure into components for manipulation or the mental rotation of a spatial configuration in short term memory, and it requires performance of serial operations, perhaps involving an analytic strategy [14]. However many researchers have noted that some of the tasks 
used to assess spatial aptitude do not require mental rotation of images. We return to this point in the Discussion section.

\subsection{Navigational Tools on the Web}

From the general background of hypertext we now move to specifics of the WWW and how browser software mediates users' interaction.

Much research has been conducted to determine what cognitive processes are involved when users interact with Web browsers. Popular Web browsers provide some tools to aid in navigation. The 'Back', 'Forward', and 'History' buttons are most commonly seen in this category. The 'Back' and 'Forward' buttons rely on the unrealistic notion of an one-dimensional path [15].

During navigation, users may employ the tools to refrain from becoming disoriented (i.e., not recognizing where they 'are' in a website) or becoming lost (i.e., not being able to easily return to the webpage they want to visit). Almost all Web browsers have such tools, and many websites have their own navigation aids. For example, breadcrumbs are commonly seen in large, hierarchical websites, such as the Yahoo! Directory. Breadcrumbs are intended to help users by providing information on their location in the website [9]. Fig. (1) shows examples of two the two types of breadcrumbs we used in the experiment on which we are reporting.

Breadcrumbs are often implemented to help website users whose first visit is not one of the webpages intended (by the website architect) for entry to the site. Breadcrumbs also facilitate revisition of previous pages if the user does not reach the current page from a search result or is not redirected from another site. The look-ahead variation of regular breadcrumbs present the part of site structure in a two-dimensional hierarchical way through the in-place expandable menu layout [16]. The two-dimensional spatial layout of menu breadcrumbs is one of the aspects that were investigated earlier [9, 16].

\subsection{Purpose of the Work}

Briefly, our goal in the studies we report here is to gain understanding of differences in the ways that people with varying levels of spatial aptitude navigate in large hierarchical websites.

In particular we are working to develop models of user cognition for use in user-centred design of better tools for using hypertext on the WWW.

Many reports of the influence of spatial aptitude on success with hypertext refer to simulated physical worlds, e.g. the 3D VRML-based information structure used by Chen [17]. However, the cognitive processes used in such simulations, and indeed in navigating in the physical world, are different from those used when processing text [18] (p. 573). This article is about the interaction of SA and success in using hypertext that is not similar to navigating in the physical world. Although we include hypermedia in our

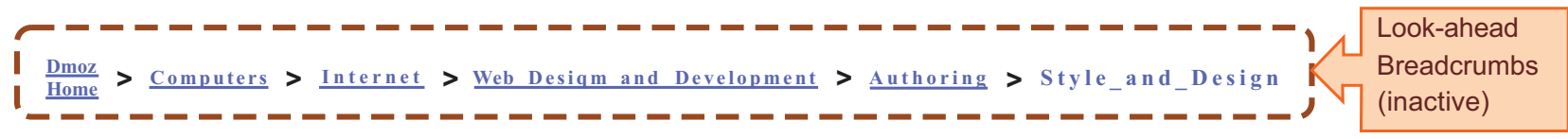

d] $\mathbf{m} \mathbf{z}$ open directory project

about dmoz $\mid \underline{\text { suggest URL }}$ update

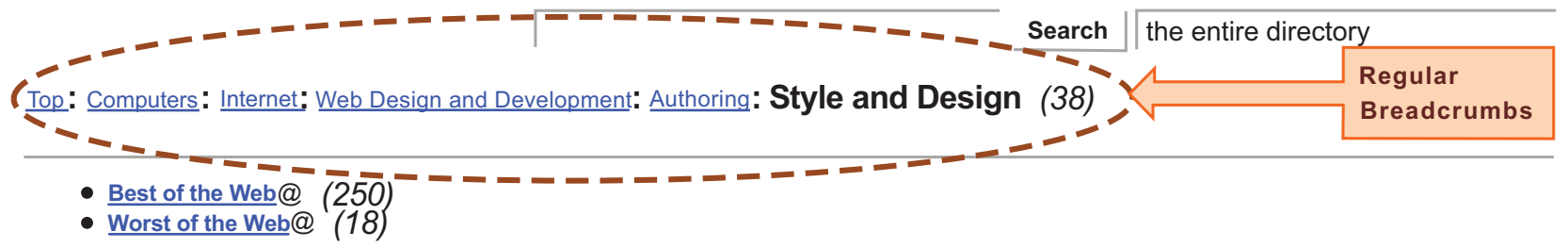

(a) Look-ahead and Regular breadcrumbs

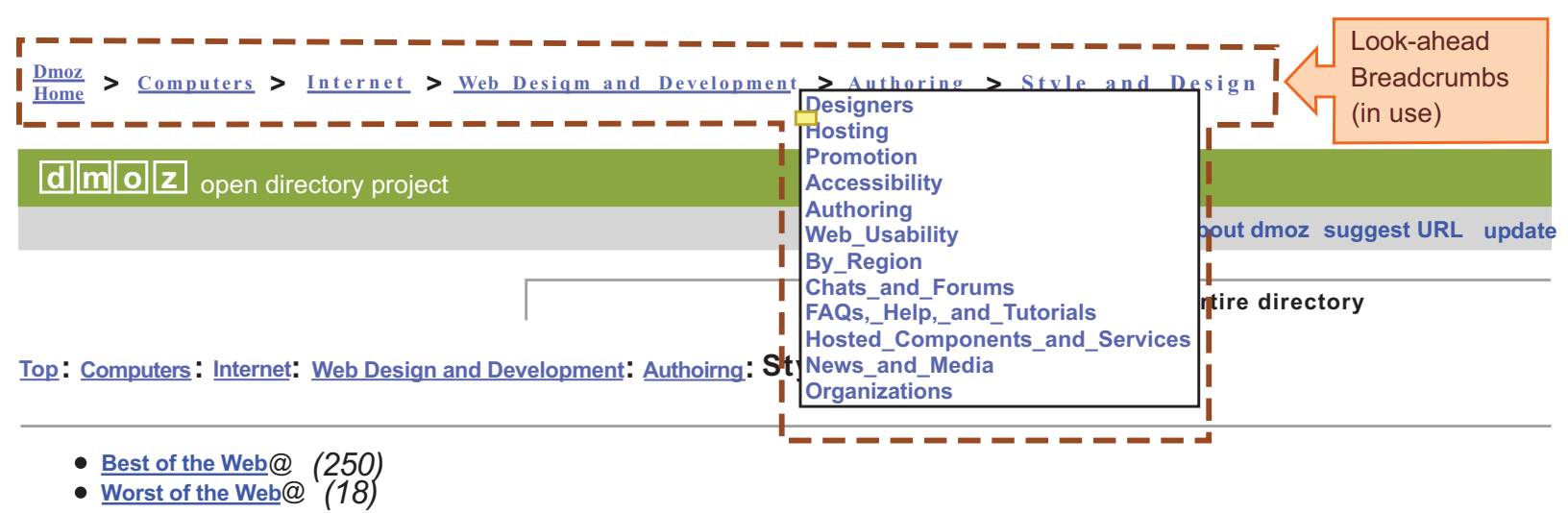

(b) An example of Look-ahead breadcrumbs in use

Fig. (1). Annotated examples of types of breadcrumbs. 
working definition of hypertext, we are studying presentations that are primarily text-based.

\section{BACKGROUND}

The work of Paivio [19] on mental processing of information demonstrated that the aptitude to encode conceptually presented information into a spatial representation in a reader's mind could help in learning and memory. It is somewhat unclear how people conceptualize the simulated environment of Web browsing and how much of that conception is spatial. However we do know that people often discuss their use of the WWW as though they were traveling through space $[20,21]$.

Dillon [22, 23] suggests that readers might form mental representations of the texts they read which combine physical and logical characteristics. $\mathrm{He}$ terms this 'information shape'. If his supposition is correct then readers likely use those models to navigate in the 'space' of text [24]. Navigation becomes especially important in hypertext, particularly where the component texts (e.g. paragraphs) are noncontiguous $[1,25]$. Before we discuss this idea further we present a brief review of key experimental results.

\subsection{Previous Key Results}

Spatial organization of information allows scanning large quantities of information with little effort. When the information units are connected topologically, any linear, two-dimensional, tree-like hierarchical or three-dimensional information layouts can be considered as spatial representation [26]. The consensus view amongst researchers in this field is that the textual can be considered spatial only if the presentation has more than one dimension $[13,27]$.

Much research reported the relation between spatial aptitude of an individual and navigation in information space [5, 28, 29]. Vicente and Williges [13] found that SA influenced the user's aptitude to navigate a large file structure. Benyon and Murray [28] found that a combination of SA and computer experience affected users' aptitude to interact with a database system. Some studies concluded that navigation performance of people with lower spatial aptitude improved by providing them with visual tool that assisted in understanding the layout of the space [27, 30]. However some studies did not support that conclusion [13, 17, 27, 30].

Some studies concluded that the navigation performance of people with lower spatial aptitude could be improved by providing them with visual tool that assisted in understanding the layout of the space [27,30]. Wiebe [31] reported that spreadsheet-like representation of nodes helped people in quick learning of relationship among nodes.

In a study by Vicente and Williges [13], participants performed navigation using both verbal and visual file systems. They used the concept of 'momentum' referring to how participants integrated and extracted information from different interfaces. The result showed that the performance difference within the participant group was not significant across the interfaces. Stanney and Salvendy [27] found that visual tools, such as two-dimensional and three-dimensional organization of information, removed the discrimination of mental models between people with lower and higher SA. They designed three structures of information representation:
(1) click-based exploration, (2) hierarchical, and (3) purely linear. They concluded that participants with lower SA could not use the first interface due to failure to create a mental representation of the information. But no difference in performance was found between participants with lower and higher SA for either of the other representations.

In studies of searching bibliographic records Allen [30] found that participants with lower visualization spatial aptitude performed better with two dimensional representations of information, however the same design features deteriorated performance of participants with high spatial visualization aptitude. Dählback and Höök [32] tested an online, hypermedia based help system and found that visual navigation aids provided help to people with lower SA. Campagoni and Ehrlich [33] reported that a good SA helped one to learn the structure of the domain quickly. In their study, participants with higher visualization SA used the top-level table of contents less frequently than participants with lower SA. Leidigh [34] only found a marginal effect of SA on the accuracy of performance when using hypertext structure maps, although a significant effect of SA on users' general satisfaction was reported.

Table 1. Distributions of Efficiency Measures

\begin{tabular}{|c|c|c|}
\hline \multirow{2}{*}{} & \multicolumn{2}{|c|}{ Spatial Ability } \\
\cline { 2 - 3 } & Lower & Higher \\
\hline \hline Range & $0.17-1.51$ & $0.00-1.41$ \\
\hline Mean & 1.05 & 0.84 \\
\hline Median & 1.07 & 0.89 \\
\hline Std. Dev. & 0.35 & 0.38 \\
\hline
\end{tabular}

\subsection{Summary of Previous Work}

It appears that spatial aptitude (SA) is an important factor in success with hypertext: some studies found that users with higher SA performed better than those with lower SA; some studies found that interface tools that led to improved performance for users with lower SA led to decreased performance for those with higher SA. Previous studies had the limitation that none of those investigated the performance of people with diverse SA during navigation in large WWW-based environment.

Readers navigate to revisit and explore parts of a text. It could well be that all readers use a form of mental representation for navigation, as hypothesized by Dillon [23], and that this representation is closely tied to the VZ factor of spatial aptitude. The apparent contradiction posed by the inverse relationship of higher SA and poor performance (and vice versa) in some studies could be due to interference between the reader's internal representational mechanism and the specific representation used in the hypertext [35].

The results of previous studies suggest that spatial aptitude somehow influences how readers perceive and use hypertexts. The applications to information systems on the WWW are obvious. If we could understand how and why these differences affect users there could be substantial 
changes to the design of websites and the experience of using the WWW: Adaptive hypermedia techniques could be used to tailor the presentation of information; Users with particular needs (perhaps researchers who now spend hours 'on' the WWW daily) would perform better with specialized training [36].

\section{HYPOTHESIS}

An early step in understanding this phenomenon is to determine how spatial aptitude affects the use of websites. We confine our interest to large hierarchically-structured websites at first (e.g. Yahoo!, Open Directory Project, Wikipedia) since they are frequently used information sources, the effects of their structure has been studied extensively in the cognitive psychology literature. Also, although they are hypertextual their structure is not erratic $[6,7]$.

We hypothesized that spatial aptitude would be a significant factor in navigation. We also hypothesized that there would be an interaction between SA and the ways breadcrumbs were presented.

\section{METHOD}

Participants navigated through a large, hierarchical website to locate the single webpage that could answer a question. In half of the four navigation tasks participants were provided with look-ahead breadcrumbs; regular breadcrumbs and the 'Back' button were available for all tasks. The navigation tasks were organized in a balanced crossover design: Each condition had four such tasks with alternating sequence of presence of regular and menu breadcrumbs along with other navigational aid in the browser. The first two tasks were specified as a practice session and the last two as the test session.

Before performing navigation tasks, each participant completed two tests to assess spatial aptitude.

\subsection{Participants}

The twenty-six unpaid volunteer participants of the experiment were graduate $(n=16)$ and undergraduate ( $n=10)$ students of Dalhousie University. Sixteen participants were from Computer Science discipline and seven were from Faculty of Science and the rest were from other disciplines. The spread of age range was from $<20$ $(n=1)$ to $41-50(n=1)$ with the age range $21-25$ years having the highest frequency (namely 12). There were nineteen male and seven female participants and all of them were familiar with the WWW environment and spend an average of 6-10 hours using the Internet each day. One participant had difficulty understanding the language of the website and hence, the result from that participant was excluded from the final analysis but was used in the range of SA scores.

\subsection{Instructions to the Participants}

Participants were neither encouraged nor discouraged to prefer any of the navigation tools. They were told about the experimental procedure. Each of them was given computerbased instructions to look for the answer in the pages and to avoid visiting external pages. The functions of the buttons in the browser toolbar were explained at the beginning of each online part of the study.

Participants were not allowed to open multiple windows of the browser and prohibited to use the search feature of the experiment websites. If the participants used the search feature or reached an external page from the site, the browser directed the request to a default page at file://external.html. Participants could easily get back to the experiment site from that page by clicking the Back button or a link titled 'Click here to go back to the last visited page'. Participants who were not done a task within twelve minutes were reminded that they could quit that task and start the next one at anytime.

An example of a typical question that participants were given was

Color $\mathrm{Me}$ On (colormeon.com) offers color-based personality assessment and related training to corporations and individuals for better understanding, compatibility and performance. Please find the webpage where Color Me On is listed in the website.

\subsection{Website and Data Extraction}

The study required a large hierarchical website where a meaningful hierarchy exists. The Open Directory Project site (http://www.dmoz.org/) is such a site. At the time of the study it contained more than 4 million site references having approximately 64,000 editors and over 590,000 categories. The webpages for this experiment are permitted for use according to the free use license of Open Directory Project. The whole site was downloaded for the experiment. Once copied, we reproduced a different version of the whole Open Directory Project site in which menu breadcrumbs were inserted in each page.

\subsection{Spatial Aptitude Tests}

In this study, spatial visualization aptitude was assessed using the paper-and-pencil based paper folding test (VZ-2) and surface development test (VZ-3) taken from the Kit of Factor-Referenced Cognitive Tests [14]. Many studies use these tests to measure the effect of spatial aptitude on success with hypertext [27, 30,37]. Furthermore, these tests have been used in cognitive psychology research [38].

A brief description of the tests is in order:

In the paper-folding test, participants were shown drawings of a rectangular piece of paper that had been folded symmetrically and then had one or more holes punched through it. The participants were then requested to identify by multiple choice where holes would appear if the paper were unfolded.

In the surface development test participants were shown flat diagrams of shapes to be folded, and of the three-dimensional shapes that would result if all of the folds had been made. The diagrams had labeled sides and straight edges. The participants were asked to list the order of sides that need to be folded to create the shapes. 
Both tests were scored with a penalty for incorrect answers that was equivalent to guessing.

\subsection{Data Acquisition}

Participants' interaction with the website (mouse movements, clicks, timing, etc.) was gathered by the customized usability testing service Uzilla. Uzilla is an instrumented Web browser that logs user interaction details such as clicks, mouse downs, mouse over and scrolling with the Internet based systems into its integrated, analysis and aggregation data collection server [39].

Fig. (2) shows an unusually demarcation between the groups in our experiment. Such a clear difference is unusual in the spatial aptitude literature - it makes our results the more striking.

Of the twenty-six participants, there were 13 participants for both Conditions. The participants were evenly partitioned into two groups: 'lower' and 'higher' by the median (21.38) of the spatial aptitude scores. However, after classification for Condition 1 there were 7 participants in the lower spatial aptitude group and 6 participants in the higher spatial aptitude group. For Condition 2, there were 6 participants in the lower spatial aptitude group and 6 participants in the higher spatial aptitude group. For this condition, one participant who faced problem in understanding the language of the website, was not considered for the classification.

\subsection{Independent Variable}

Spatial aptitude, the independent variable was measured as the sum of scores on the VZ-2 and VZ-3 tests. Answers were corrected for guessing following the standard procedure described in the Manual and instructions given to the participants [14]. As there are no global standards for those tests, the results are always compared within the participant population.

\subsection{Dependent Variables}

Speed was measured as the number of seconds to complete a task. Lower values represent faster speed.

Accuracy was 1 if and only if the participant completed the task successfully, and 0 otherwise. A single accuracy measure was calculated per group per task. The measure was the quotient of number of times participants completed the task accurately and total number of participants in the group.

Lostness is a scale composed of the number of unique and total pages visited by the participant compared to the optimal number of page visits required to complete the task [40]. In this study, the optimal number refers to the minimal number of page views required for the task. Smith [40] described an user as definitely 'lost in a hyperspace' if the lostness score was greater than 0.5 and any score less than 0.4 as 'not lost'.

Otter and Johnson [41] suggest two other measure for this variable. One of those measures (the link-weighted approach) is identical for our design to Smith's [40] approach. We abandoned the second (so called mental models) measure after we found serious problems with it during pilot testing.
Efficiency deals with the total number of page views versus the minimal or optimal number of page views and the accuracy of task completion. It is correlated with lostness but not perfectly, as users with the same lostness may vary in efficiency [40]. The lower the value, the greater the level of efficiency.

Preference. Participants differed based on their preference of selecting navigation tool. For this measure, the percentage of clicks on the Back button with respect to total clicks was calculated. The goal of this analysis was to measure users preference for the Back button (which was available for all webpages but only allowed single steps) versus specific tools available in a specific website and for a specific task.

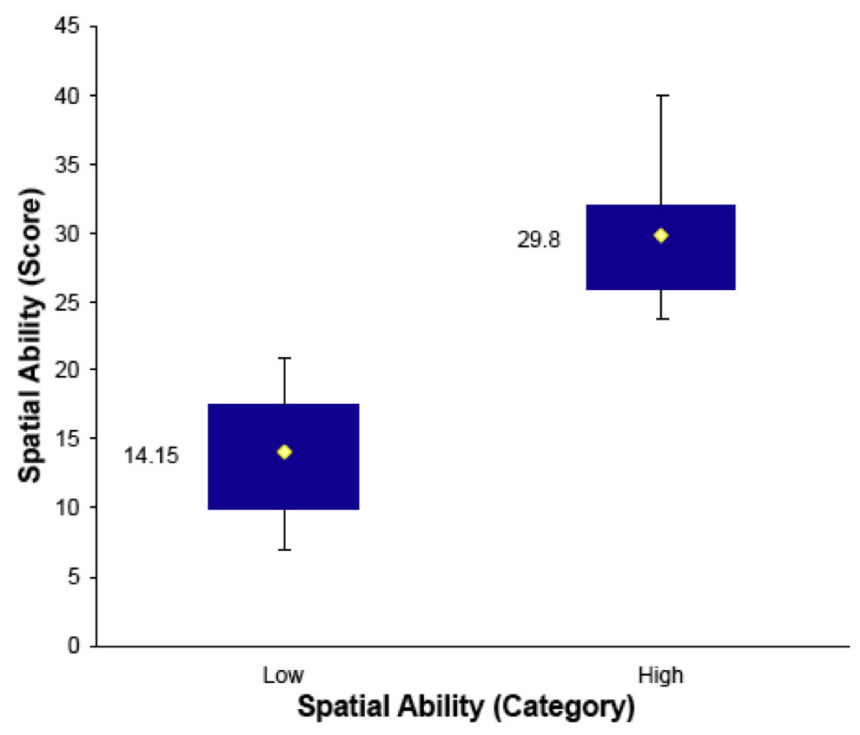

Fig. (2). Distribution of spatial aptitude scores (boxes depict 25th $75^{\text {th }}$ percentile, whiskers depict range).

\section{RESULTS}

We applied two-way repeated measure analyses of variance (ANOVAs) to our data. The analysis used breadcrumb type as the within-subject factor and spatial aptitude as the between-subject factor. No interaction effects were found in any of the analyses involving SA. We present data about the SA factor and the various measures described above.

1. Although participants with higher spatial aptitude seemed to be faster than participants with lower levels (463.6 vs. 525.2 sec.), the difference was not significant.

2. The apparent difference in lostness for participants with higher spatial aptitude ( 0.75 on average) and participants with lower levels ( 0.81 on average), the difference was not significant.

3. Participants with higher spatial aptitude were more efficient than those with lower levels ( $\mathrm{df}=1 ; F=$ 4.38; $\operatorname{Pr}>F=0.0486$ ). Descriptive statistics for both groups of participants are shown in Table 1 . The corresponding critical values and least square means are in Table 2 . 
4. Participants with higher spatial aptitude used the Back button marginally less than those with lower aptitude $(\mathrm{df}=1 ; F=4.16 ; \operatorname{Pr}>F=0.054 ; \mathrm{df}=21 ; \mathrm{t}=$ 2.12; $\operatorname{Pr}>|t|=0.0462)$. Critical values for both groups are presented in Table $\mathbf{3}$.

5. There was no significant difference between the overall accuracy scores for participants in the higher and lower spatial aptitude categories $(0.8$ and 0.5 respectively). Participants with higher spatial aptitude were more successful than participants with lower aptitude in terms of the overall accuracy score of the group.

Table 2. Effect of Spatial Aptitude on Efficiency

\begin{tabular}{|c|c|c|c|c|}
\hline \multirow{2}{*}{ Category } & \multicolumn{2}{|c|}{ Spatial Ability Scores } & \multirow{2}{*}{$\boldsymbol{t}$ Value } & \multirow{2}{*}{$\operatorname{Pr}>|\boldsymbol{t}|$} \\
\cline { 2 - 3 } & Range & LS Mean & & \\
\hline \hline Lower SA & $0.91-1.19$ & 1.05 & 15.46 & $<0.0001$ \\
\hline Higher SA & $0.70-0.99$ & 0.85 & 11.99 & $<0.0001$ \\
\hline
\end{tabular}

Table 3. Navigational Tool Preference by SA Category

\begin{tabular}{|c|c|c|c|c|}
\hline \multirow{2}{*}{ Category } & \multicolumn{2}{|c|}{$\begin{array}{c}\text { Use of Back Button } \\
\text { (Compared to Breadcrumbs) }\end{array}$} & \multirow{2}{*}{$\boldsymbol{t}$ Value } & \multirow{2}{*}{$\operatorname{Pr}>|\boldsymbol{t}|$} \\
\cline { 2 - 4 } & Range & LS Mean & & \\
\hline \hline Lower SA & $15.1-26.0$ & $20.6 \%$ & 7.90 & $<0.0001$ \\
\hline Higher SA & $7.3-18.5$ & $12.9 \%$ & 4.78 & 0.0001 \\
\hline
\end{tabular}

\section{DISCUSSION}

The analysis in the previous section revealed that spatial aptitude had a significant main effect on the efficiency: Participants with higher SA were more efficient than participants with lower SA. Participants with higher SA used the Back button significantly less than participants with lower SA. No main effect was detected for either speed or lostness measures.

Further analysis showed that, in all task arrangements, participants with higher spatial aptitude had higher overall accuracy scores than participants with lower aptitude. However, due to the nature of these measures, no statistical analysis was done but the results can be taken as informal yet guiding towards future prediction.

\subsection{Importance of $\mathrm{VZ}$ Subfactor}

These outcomes support the conclusions of other studies that SA influences navigation performance in information space. It also strongly suggests that the VZ subfactor is responsible for the effect.

\subsection{Spatial Components of the Interface}

Ekstrom et al. [14] note that earlier research found that tests for the VZ factor did not require participants to fully visualize images in order to correctly answer the test questions. Test takers need to recognize fundamental spatial characteristics (such as axes of symmetry) and reason using them but they do not need to perform mental rotation of images. This fact is of great importance in interpreting the results of our study as it could explain the connection between test results that use images and success with websites that have virtually no images. It further suggests that participants with higher SA are better able to interpret and use non-spatial tools that represent structure and other aspects necessary for navigation. To recap part of Section 2: people can be trained to improve their SA, and there is some evidence that tools which are not spatially-oriented may be more effective for the majority of WWW users. Taken together all of this indicates that navigation on the WWW could be improved by use of appropriate non-graphical aids, and that training in recognizing spatially-oriented thinking could be a boon to people who must use the WWW to answer complex queries frequently.

This study also investigated the relationship between the spatial layout of breadcrumbs and spatial aptitude of people. The spatial layout of experimental breadcrumbs did not affect the participants with higher and lower aptitude, which might have indicated that breadcrumbs can be accepted as a navigation tool for people with wide variety of spatial aptitudes.

\section{SUMMARY OF EXPERIMENT}

This work has explored the influence of innate spatial visualization aptitude in navigation of large hierarchical websites with novel navigational aids. The outcome has underlined the necessity of further efforts and hinted what it will take to build widely acceptable navigation tools and interfaces for people with diverse individual differences. Even though there was no difference in time taken to complete the (realistic) tasks, readers with higher SA used different techniques to navigate the website than their counterparts with lower SA.

There is clear evidence of at least two sub-factors of spatial aptitude that have substantial effect on success with hypertext in one of the simplest hypertext conditions (namely, hierarchy) [7]. Experimental avenues leading from these results are clear. Practical developments that are likely to follow from such experiments are in ways that software (browsers) and websites can be adapted to suit particular navigational styles. We do not completely reject the notion that users can be trained to work with information systems that are not ideally suited to their cognitive style, however we expect that it will be easier and more profitable to adapt systems to people rather than vice versa. We do not completely reject the notion that users can be trained to work with information systems that are not ideally suited to their cognitive style, however we expect that it will be easier and more profitable to adapt systems to people rather than vice versa.

\section{FOLLOW-UP STUDIES}

The study reported above can be extended into several directions. The various components of possible work include spatial aptitude and broader perspective of human cognition and navigation metaphor of the World Wide Web. Some independent parameters appear to affect SA such as gender, age, personality factors and other various cognitive styles [12]. It may be useful to include these aspects in future studies. 


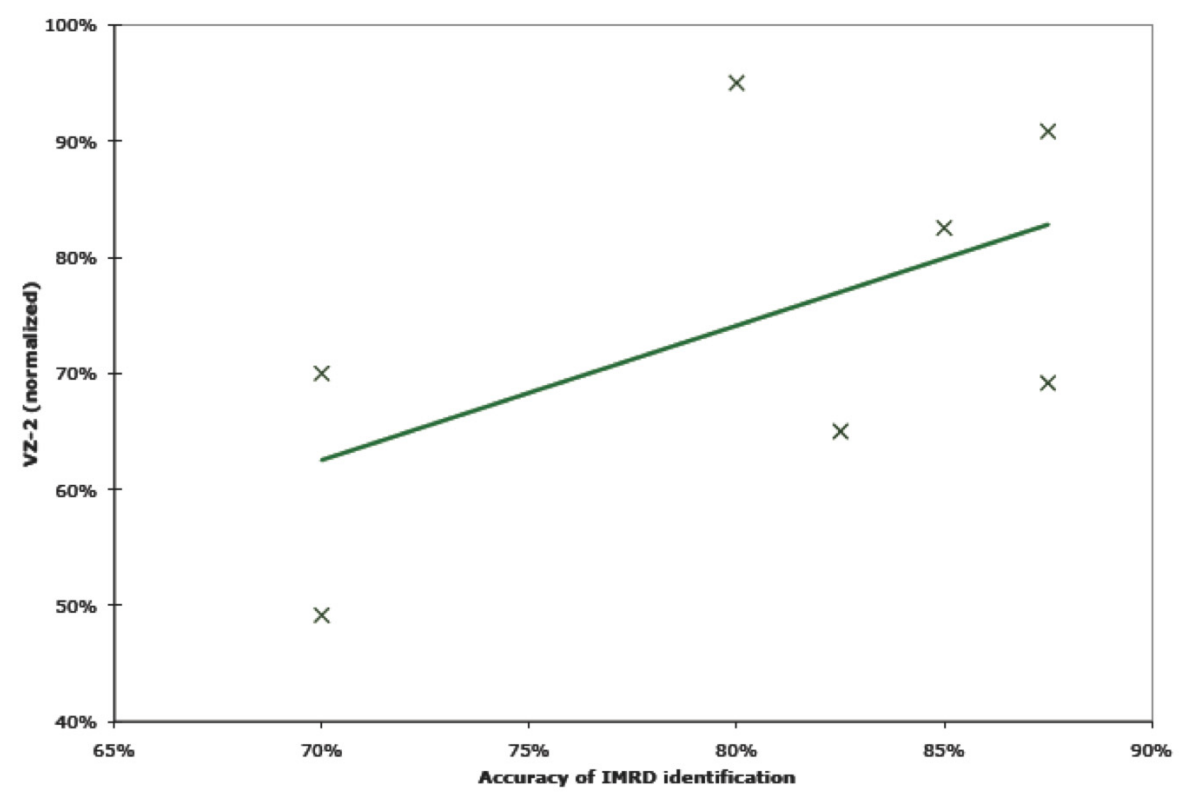

Fig. (3). Linear correlation of VZ-2 score and accuracy of identification of location of passages within a scholarly work by expert participants.

It is especially apt to examine the basis for Dillon's concept of information shape, which we descried briefly in Section 2. We have begun to reproduce the experiment he and Schaap used to test experts on their categorization of logical units within human factors literature [42] in combination with tests of SA. Following the method described in their publications we have conducted nine tests, from which seven participants were identified as experts. We have not completely analysed that data yet and are hoping to recruit more experts. In the meantime however we can report that a slightly linear $(r=.55)$ correlation appears to exist between aptitude for the VZ-2 (paper folding task) factor and categorization of logical units. The data and result are illustrated in Fig. (3). According to McBurney and White [43] that correlation is considered significant (with $p<0.10$ ) for an exploratory study such as we are reporting. We are encouraged to continue exploring the complex relationship between spatial aptitude and both perception of information shape and success with hypertext. Note for example that the multiple values at the $70 \%$ and $88 \%$ passage identification accuracy levels suggests that there is more than a single factor at work.

The first author is beginning a much larger and more detailed version of the study reported in Section 4 to try to determine the details of the interaction between spatial ability and success with hypertext. The goal of that work is firstly to gain a better understanding of the nature of the effect, and secondly to point the way to the development of practical tools and recommendations to improve the use of the WWW.

\section{ACKNOWLEDGMENTS}

The authors gratefully acknowledge the suggestion to investigate breadcrumbs for navigation, and ongoing support in pursuing the investigation, from Keith Instone. Menu breadcrumbs were conceived and created by Hui Teng. We are grateful to Brad MacPhee for his help with the program that inserted the breadcrumbs. We are grateful to Jack Duffy and David Hamilton for advice on experimental design and statistical analysis. This work is supported by a NSERC Discovery grant. Groundwork for this project was laid by Khalid Najia, Jason Satel, and Harman Clair. Approval was secured from the Dalhousie University Office of Research Ethics Administration to conduct the experiments described herein.

\section{REFERENCES}

[1] Charney D. The effect of hypertext on processes of reading and writing. In: Selfe CL, Hilligoss S, Eds. Literacy and computers: the complications of teaching and learning with technology. New York, NY: The Modern Language Association of America 1994; pp. 238-63. Available from: http://www.cwrl.utexas.edu/\%7 Echarney/homepage/Articles/Charney_hypertext.pdf

[2] Wright P. Cognitive overheads and prostheses: some issues in evaluating hypertexts. Hypertext '91 Proceedings. ACM. Assoc Comput Machine 1991; 1-12. Available from: http://doi.acm. org/10.1145/122974.122975

[3] Nielsen J. The matters that really matter for hypertext usability. In: Meyrowitz N, Ed. Hypertext '89 Proceedings. ACM. Pittsburgh, PA Assoc Comput Machine 1989; 239-48. Available from: http://doi.acm.org/10.1145/74224.74244

[4] Benyon D, Höök K. Navigation in information spaces: Supporting the individual. Proceedings of human-computer interaction: INTERACT' 97. Chapman and Hall 1997; pp. 39-46.

[5] Chen C, Rada R. Interacting with hypertext: a meta-analysis of experimental studies. Hum Comput Interact 1996; 11(2): 125-56. Available from: http://dx.doi.org/10.1207/s15327051hci1102_2

[6] Larson K, Czerwinski M. Web page design: implications of memory, structure, and scent for information retrieval. In: Karat CM, Lund A, Coutaz J, Karat J, Eds. Making the impossible possible: CHI 98 human factors in computing systems conference proceedings. New York, NY: ACM Press 1998; pp. 25-32. Available from: http://doi.acm.org/10.1145/274644.274649

[7] Brockmann RJ, Horton W, Brock K. From database to hypertext via electronic publishing: an information odyssey. In: Barrett E, Ed. The society of text: hypertext, hypermedia, and the social construction of information. Cambridge, MA: The MIT Press 1989; pp. 162-205.

[8] Parunak HVD. Ordering the information graph. In: Berk E, Devlin J, Eds. Hypertext/Hypermedia Handbook. New York, NY: Intertext Publications 1991; pp. 299- 325.

[9] Blustein J, Ahmed I, Instone K. An evaluation of menu breadcrumbs for the WWW. HYPERTEXT '05: Proceedings of the 
sixteenth ACM conference on hypertext and hypermedia. New York, NY: ACM Press 2005; pp. 202-4. Available from: http://doi.acm.org/10.1145/1083356.1083394

[10] Lohman DF, Kyllonen PC. Individual differences in solution strategy on spatial tasks. In: Dillon RF, Schmeck RR, Eds. Individual differences in cognition. Hum Comput Interact Academic Press Inc. 1983; vol. 1: pp. 105-35.

[11] Carroll JB. Human cognitive abilities: a survey of factor-analytic studies. NY: Cambridge University Press 1993.

[12] Sjölinder M. Individual differences in spatial cognition and hypermedia navigation. Towards a framework for design and evaluation of navigation in electronic spaces. Swedish Inst Comput Sci 1996; 61-72. Available from: http://www. sics.se/humle/proje cts/persona/web/littsurvey/ch5.pdf

[13] Vicente KJ, Williges RC. Accommodating individual differences in searching a hierarchical file system. Int J Man Mach Stud 1988; 29(6): 647-68.

[14] Ekstrom RB, French JW, Harman HH, et al. Eds. Kit of factorreferenced cognitive tests. Princeton, NJ: Educational Testing Service 1976; p. 19.

[15] Jones S, Cockburn A. A study of navigational support provided by two World Wide Web browsing applications. HYPERTEXT ' 96 Proc seventh ACM conf Hypertext 1996; pp. 161-9. Available from: http://doi.acm.org/10.1145/234828.234844

[16] Teng H. Location breadcrumbs for navigation: an exploratory study [Masters]. Dalhousie University Faculty of Computer Science. Halifax: NS, Canada 2004.

[17] Chen C. Individual differences in a spatial-semantic virtual environment. J Am Soc Inf Sci 2000; 51(6): 529-42. Available from: http://dx.doi.org/10.1002/(SICI)1097-4571(2000)51:6<529:: AID-ASI5>3.0.CO;2-F

[18] Kolb B, Wishaw IQ. Fundamentals of human neuropsychology. NY: W. H. Freeman 1996.

[19] Paivio A. Mental Representations: A dual coding approach. Oxford University Press: UK 1986.

[20] van Hooijdonk C, Maes A, Ummelen N. I have been here before': An investigation into spatial verbalizations in hypertext navigation. Inf Des J 2006; 14(1): 8-21.

[21] Maglio PP, Matlock T. The conceptual structure of information space. In: Munro AJ, Höök K, Benyon D, Eds. Social navigation of information space. Computer supported cooperative work. Springer-Verlag: London Limiteds 1999; pp. 155-73.

[22] Dillon A. Readers' models of text structures: the case of academic articles. Int J Man Mach Stud 1991; 35: 913-25.

[23] Dillon A. Spatial-Semantics: How users derive shape from information space. J Am Soc Inf Sci 2000; 51(6):521-8. Available from: http://dx.doi.org/10.1002/(SICI)1097-4571(2000)51:6<521:: AID-ASI $>3.0 . \mathrm{CO} ; 2-5$

[24] Spence R. A framework for navigation. Int J Hum Comput Stud 1999; 51(5): 919-45. Available from: http://dx.doi.org/10.1006/ ijhc. 1999.0265

[25] Bernstein M. Patterns of hypertext. In: Grønæbk K, Mylanos E, Shipman FM III, Eds. Hypertext '98: The proceedings of the ninth ACM conference on hypertext and hypermedia. Pittsburgh, PA: ACM SIGLINK and ACM SIGIR 1998; pp. 21-9. Available from: http://doi.acm.org/10.1145/276627.276630

[26] Mark DM. 7. In: Spatial Representation: A Cognitive View. $2^{\text {nd }}$ ed. USA, Wiley \& Sons Inc. 1999; pp. 81-9.
[27] Stanney KM, Salvendy G. Information visualization: Assisting low spatial individuals with information access tasks through the use of visual mediators. Ergonomics 1995; 38(6): 1184-98.

[28] Benyon D, Murray DM. Adaptive systems: From intelligent tutoring to autonomous agents. Knowl Based Syst 1993; 6(4): 197219. Available from: http://dx.doi.org/10.1016/0950-7051(93) 90012-I

[29] Egan D. Individual differences in human-computer interaction. In: Helander MG, Landauer TK, Prabhu PV, Eds. Handbook of human- computer interaction. $2^{\text {nd }}$ ed. Amsterdam: Elsevier Science Publishers 1998; pp. 543-68.

[30] Allen B. Individual differences and the conundrums of usercentered design: two experiments. J Am Soc Inf Sci 2000; 51(6): 508-20. Available from: http://dx.doi.org/10.1002/(SICI)10974571(2000)51:6<508::AID-ASI3>3.0.CO;2-Q

[31] Wiebe EN. A review of dynamic and static visual display techniques. Engineering design graphics division of the american society for engineering education. Mid-Year Meeting; 1991 p. 6. Available from: http://www4.ncsu.edu/\%7Ewiebe/ articles/ani1991.pdf

[32] Dählback N, Höök K, Sjölinder M. Spatial cognition in the mind and in the world: The case of hypermedia navigation. The eighteenth annual meeting of the cognitive science society. San Diego, CA, University of California 1996; pp. 195-200.

[33] Campagoni F, Ehrlich K. Information retrieval using a hypertextbased help system. ACM Trans Inf Syst 1989; 7(8): 271-91.

[34] Leidigh PM. The relationship between cognitive styles and mental maps in hypertext assisted learning [PhD]. Virginia Commonwealth University 1992.

[35] McKendree J, Reader W, Hammon N. The "Homeopathic Fallacy" in learning from hypertext. Interactions 1995; ii(3): Available from: http://doi.acm.org/10.1145/208666.208687

[36] Upitis R, Dearden R, Inkpen K, et al. Weird-a-gons and other folded objects: the inuence of computer animation, paper models, and cooperative mediation on spatial understanding. University of British Columbia; 1994 TR-94-30. Available from: https://www.cs. ubc.ca/nest/egems/reports/3dpaper.ps

[37] Blustein J, Satel J. Spatial Ability and information shape: When do individual differences matter. In: Shipman FM III, Rosenberg J, Eds. Third Workshop on Spatial Hypertext 2003; p. 41. http://www.cs.dal.ca/research/techreports/2003/CS-2003-11.shtml

[38] Downing RE, Moore JL, Brown SW. The effects and interaction of spatial visualization and domain expertise on information seeking. Comput Hum Behav 2005; 21(2): 195-209. Available from: http://dx.doi.org/10.1016/j.chb.2004.03.040

[39] Edmonds A. Uzilla: A new tool for web usability testing. Behav Res Methods Instrum Comput 2003; 35(2): 194-201.

[40] Smith PA. Towards a practical measure of hypertext usability. Interact Comput 1996; 8(4): 365-81. Available from: http://dx.doi.org/10.1016/S0953-5438(97)83779-4

[41] Otter M, Johnson J. Lost in hyperspace: metrics and mental models. Interact Comput 2000; 13: 140. Available from: http://dx.doi.org/10.1016/S0953-5438(00)00030-8

[42] Dillon A, Schaap D. Expertise and the perception of shape in information. J Am Soc Inf Sci 1996; 47(10): 786-8. Available from: http://dx.doi.org/10.1002/(SICI)10974571(199610)47:10<786:: AID-ASI7>3.0.CO;2-Z

[43] McBurney DH, White TL. Research methods. $6^{\text {th }}$ ed. Toronto: Wadsworth 2004. 